\title{
Inhibitory control elicited by physical activity and inactivity stimuli: an EEG study
}

\section{RUNNING HEAD: PHYSICAL INACTIVITY AND INHIBITORY CONTROL}

\author{
Boris Cheval ${ }^{1,2^{*}}$, Daniel A. R. Cabral ${ }^{3}$, Marcos Daou ${ }^{4}$, Mariane F. B. Bacelar ${ }^{3}$, Juliana O. \\ Parma $^{3}$, Cyril Forestier ${ }^{5}$, Dan Orsholits ${ }^{6}$, Silvio Maltagliati ${ }^{7}$, David Sander ${ }^{1,2}$, Matthieu P. \\ Boisgontier $^{8,9}$, Matthew W. Miller $3,10^{*}$
}

${ }^{1}$ Swiss Center for Affective Sciences, University of Geneva, Geneva, Switzerland

${ }^{2}$ Laboratory for the Study of Emotion Elicitation and Expression (E3Lab), Department of Psychology, FPSE, University of Geneva, Geneva, Switzerland

${ }^{3}$ School of Kinesiology, Auburn University, USA

${ }^{4}$ Department of Kinesiology, Coastal Carolina University, USA

${ }^{5}$ Laboratoire Motricité, Interactions, Performance, MIP - EA4334, Le Mans Université, Le Mans, France

${ }^{6}$ Swiss NCCR “LIVES - Overcoming Vulnerability: Life Course Perspectives", University of Geneva, Switzerland

${ }^{7}$ Univ. Grenoble Alpes, SENS laboratory, Grenoble, France

${ }^{8}$ School of Rehabilitation Sciences, Faculty of Health Sciences, University of Ottawa, Ottawa, ON, Canada

${ }^{9}$ Bruyère Research Institute, Ottawa, ON, Canada

${ }^{10}$ Center for Neuroscience, Auburn University, USA

*Corresponding authors: 301 Wire Rd., Kinesiology Bldg., Auburn University, AL, USA; mwm0024@auburn.edu; @matt_pep (M. W. Miller); Campus, Biotech, Chemin des mines 9, 1202, Genève, Switzerland; boris.cheval@unige.ch; @ChevalBoris (B. Cheval).

Citation: Cheval, B., Cabral, A.R., Daou, M., Bacelar, M.F.B., Parma, O., Forestier, C., Orsholits, D., Maltagliati, S., Sander, D., Boisgontier, M.P., Miller, M.W. (2021). Inhibitory control elicited by physical activity and inactivity stimuli: an EEG study

All the authors listed in the by-line have agreed to the by-line order and have approved this version of the manuscript.

The first author can be reached on Twitter@chevalboris 


\begin{abstract}
The theory of effort minimization in physical activity (TEMPA) argues that individuals have an automatic attraction toward effort minimization. To engage in a physically active behavior, this automatic attraction needs to be overridden by controlled processes. However, direct evidence showing that inhibitory control is required to avoid effort minimization is lacking. Here, we used go/no-go tasks and electroencephalography (EEG) to assess the neural correlates of inhibitory control associated with visual stimuli depicting physical inactivity, physical activity, or that were neutral in 50 healthy young adults. The N2 event-related potential (ERP) component amplitude was used as a physiological index of inhibitory control. Results showed significant two-way interactions between the type of trials (i.e., go vs. no-go trials) and the type of stimuli on N2, revealing a significantly more pronounced no-go effect (i.e., higher N2 in no-go relative to go trials) for neutral and physical inactivity stimuli compared with physical activity stimuli. Simple tests further revealed that N2 amplitude was more negative in no-go than go trials for neutral stimuli $(\mathrm{b}=-.91 \mu \mathrm{V}, 95 \% \mathrm{CI}=-1.42$ to $-.40 \mu \mathrm{V}, \mathrm{p}<.001)$ and for stimuli depicting physical inactivity $(\mathrm{b}=-.58 \mu \mathrm{V}, 95 \% \mathrm{CI}=-1.08$ to $-.08 \mu \mathrm{V}, p=.025)$. By contrast, we found no evidence of significant differences in N2 amplitude between no-go and go trials for stimuli depicting physical activity $(\mathrm{b}=.20 \mu \mathrm{V}, 95 \% \mathrm{CI}=-1.08$ to $-.08 \mu \mathrm{V}, p=.445)$. These findings provide evidence that inhibiting responses to physical inactivity stimuli requires significantly higher inhibitory control than inhibiting responses to physical activity stimuli. The study pre-registration form can be found at https://doi.org/10.17605/OSF.IO/RKYHB.
\end{abstract}

Keywords: electroencephalography; response inhibition; go/no-go; energetic cost minimization; physical activity 


\section{Inhibitory control elicited by physical activity and inactivity stimuli: an EEG study}

Physical inactivity is a widespread and consequential health issue, with over a quarter of the world's adults being physically inactive (Bull et al., 2020; Guthold et al., 2018), leading to approximately 5 million deaths (Lee et al., 2012; WHO, 2020) and costing 67.5 billion international dollars each year (Ding et al., 2016). Paradoxically, most people are aware that physical inactivity poses a serious threat to their health and intend to be physically active. For example, a survey of United States adults revealed that $89 \%$ believed physical inactivity to be a very important or somewhat important health risk (Martin et al., 2000). Likewise, a survey of Canadian adults showed that $94 \%$ have at least a moderate-to-strong intention to be physically active (Canadian Fitness and Lifestyle Research Institute, 2018). As people seem to be aware of the health consequences associated with physical inactivity and have the intention to be active, why do many individuals fail to translate this intention into action?

Based on a neuropsychological approach to physical effort (Bernacer et al., 2019; KleinFlügge et al., 2016) anchored in an evolutionary perspective (Cheval, Radel, et al., 2018; Lee et al., 2016; Lieberman, 2015; Speakman, 2019), the recent theory of effort minimization in physical activity (TEMPA) proposes that an automatic attraction toward physical inactivity may explain this failure to engage in physical activity (Cheval \& Boisgontier, in press). The theory is supported by experimental evidence (see Cheval, Radel, et al., 2018 for a review) demonstrating that physically inactive opportunities can act as temptations interfering with physical activity goals (Cheval et al., 2017; Rouse et al., 2013). Additional evidence further suggested that a strong automatic approach tendency toward stimuli depicting physically inactive behaviors predicted lower engagement in a non-volitional physical activity (i.e., spontaneous strength invested in a 
handheld dynamometer) (Cheval et al., 2014) and explained why individuals intending to be physically active fail to turn this intention into action (Cheval et al., 2015).

If people are automatically attracted to physical inactivity, then cognitive resources may be of particular importance to counteract this attraction and may thereby favor engagement in physical activity. Epidemiological studies support this corollary of TEMPA (Cheval, Orsholits, et al., 2020; Cheval et al., 2019; Daly et al., 2015; Lindwall et al., 2012; Sabia et al., 2017; Snowden et al., 2011; Young et al., 2015). For example, older adults with more cognitive resources (e.g., higher delayed recall and verbal fluency performance) maintained physical activity during aging better than those with fewer resources (Cheval, Orsholits, et al., 2020). Besides epidemiological studies, experimental research has been conducted to examine people's spontaneous reactions toward visual stimuli depicting physical activity and physical inactivity, as these automatic reactions have been thought to explain people's engagement in physical activity (Cheval, Miller, et al., 2020; Conroy et al., 2010; Moffitt et al., 2019; Rebar et al., 2015). These experimental data suggest cognitive resources are important for avoiding physical inactivity stimuli (Cheval, Daou, et al., 2020; Cheval, Tipura, et al., 2018). For example, individuals exhibited greater brain activity associated with conflict monitoring and inhibitory control when avoiding physical inactivity stimuli, compared to physical activity stimuli in an approach-avoidance task (Cheval, Tipura, et al., 2018). Similarly, to more accurately assess response inhibition, Cheval, Daou, et al. (2020) had participants complete a go/no-go task wherein they were asked to avoid making a button-press response on trials with physical inactivity images (no-go trials) and to respond on trials with physical activity images (go trials) in an inhibit physical inactivity condition. Participants were asked to do the opposite in an inhibit physical activity condition. Results showed participants made more commission errors (i.e., failure to refrain a response to a "no-go" stimulus) in the inhibit 
physical inactivity condition, meaning participants were more likely to erroneously respond to a no-go trial if the image depicted physical inactivity, compared to physical activity ( $23 \%$ for stimuli depicting physical activity vs. $30 \%$ for stimuli depicting physical inactivity). This finding is consistent with the idea that inhibiting responses to physical inactivity stimuli taxed cognitive resources, specifically inhibitory control, to a greater extent than inhibiting responses to physical activity stimuli. This study also recorded participants' electroencephalography (EEG) while they performed the task to provide more direct evidence about whether withholding responses to physical inactivity stimuli requires greater inhibitory control. Thus, the analysis of EEG data will be the focus of the present manuscript.

EEG can provide a physiological index of the inhibitory control demanded to avoid stimuli in go/no-go paradigms. The N2 component of the event-related potential (ERP) is the most common EEG measure used to gauge inhibitory control (Folstein \& Van Petten, 2008). The N2 is a negative deflection in the ERP, usually seen $200-300 \mathrm{~ms}$ following stimulus onset and is maximal at frontocentral electrode sites. N2 amplitude is enhanced for stimuli that require overriding a prepotent response, which helped establish N2 as an index of inhibitory control. Notably, the N2 has been used to index the inhibitory control demanded in a go/no-go paradigm related to other health behaviors. Specifically, Carbine et al. (2017) had participants complete a go/no-go task wherein participants were asked to inhibit responses on trials with high-calorie (e.g., ice cream, hamburgers) images (no-go trials) and to respond on trials with low-calorie (e.g., broccoli, apples) images (go trials) in an inhibit high-calorie condition, and do the opposite in an inhibit low-calorie condition. Results showed the N2 was larger for no-go trials than go trials in the avoid high-calorie condition but not the avoid low-calorie condition, suggesting that 
withholding responses from high-calorie food images required higher inhibitory control than withholding responses from low-calorie food images.

The aim of the present study was to assess the neural mechanisms underlying the greater difficulty in withholding responses to physical inactivity stimuli compared with physical activity stimuli. Healthy young individuals completed a go/no-go task presenting physical activity and inactivity stimuli and a control condition presenting neutral stimuli (Cheval, Daou, et al., 2020). We used N2 as an indicator of inhibitory control (Folstein \& Van Petten, 2008). Based on the TEMPA (Cheval \& Boisgontier, in press; Cheval, Radel, et al., 2018), we hypothesized that, relative to inhibiting responses to physical activity stimuli, N2 should be higher when inhibiting responses to physical inactivity stimuli (i.e., higher demands on inhibitory control) (H1). Moreover, because inhibitory control is thought to play a key role in explaining individuals' ability to counteract the automatic attraction toward physical inactivity (Cheval, Orsholits, et al., 2020), we hypothesized that these cortical outcomes should be less pronounced when the usual level of physical activity increases (H2). Finally, we repeated all analyses using the recently established, but less widely used, frontal midline theta $(\mathrm{FM} \theta)$ EEG measure as a secondary outcome variable. FM $\theta$ reflects neuronal oscillations between 4 and $8 \mathrm{~Hz}$ recorded at frontocentral electrode sites and its power increases when inhibitory control is required, such as when overriding a prepotent response (Cavanagh \& Frank, 2014). Similar to the N2, FM $\theta$ has been used to index inhibitory control demanded in a go/no-go paradigm related to food stimuli (van de Vijver et al., 2018). As such, consistent with the N2 hypotheses, we predicted that participants should exhibit greater FM $\theta$ when inhibiting responses to physical inactivity stimuli compared with physical activity stimuli (H3), and that these effects should be moderated by participants' usual physical activity level (H4).

\section{Methods}


Study preregistration, materials, data, and statistical analysis scripts can be found at https://doi.org/10.17605/OSF.IO/RKYHB.

\section{Participants}

Sample size was determined with a power calculation using $\mathrm{G}^{*}$ Power 3.1.9.2 (Faul et al., 2007). We used the ANOVA: repeated-measures, within factors statistical test; set $\alpha=.05$ and 1- $\beta$ $=.95$; set groups $=1$; set measures=2: (1) trial (go/no-go) and (2) condition (inhibit physical activity/ inhibit physical inactivity); and set nonsphericity $\varepsilon=1$. We based our effect size on Carbine et al. (2017) given the similarities between their study and ours (e.g., both studies used a go/no-go paradigm wherein participants had to inhibit stimuli related to health-related behaviors and used N2 amplitude as the dependent variable). Carbine et al. reported a Condition $\mathrm{x}$ Trial interaction with $\eta_{\mathrm{p}}^{2}=.26$, which we rounded down to .20 . The power calculation estimated a required $N=55$, which we rounded up to 60 to account for lost data (e.g., due to poor EEG recording).

Participants, who were the same as for the behavioral study (Cheval, Daou, et al., 2020), were recruited via the Auburn University (USA) College of Education SONA participant recruitment system and by word-of-mouth. To sign up for the study, participants had to be 19 - 30 years old and free from: (1) physical impairment that makes physical activity difficult; (2) color blindness; (3) sensitivity/allergies to lotions or cosmetics; and (4) neurological impairment. Participants were offered course credit for their participation when possible. Participants were excluded if they had a history of psychiatric, neurological, or severe mental disorders, or were taking psychotropic medication or illicit drugs at the time of the study. This led to the removal of three participants. We also excluded five participants with a weak intention to be physically active (score $<5$ on a 10-point scale asking about physical activity intentions) because we were interested in examining whether an automatic attraction toward physical inactivity demands inhibitory 
control in people who intend to be physically active, who constitute the majority of the population (Canadian Fitness and Lifestyle Research Institute, 2018; Martin et al., 2000). Two additional participants were removed due to problems with data collection. Thus, the final sample was $N=$ 50 , resulting in a power of $94 \%$, keeping the other inputs constant in the power calculation (Faul et al., 2007).

\section{Procedures}

Participants gave written consent to a protocol approved by Auburn University's institutional review board. Then, they completed a questionnaire about some potential confounding variables (i.e., hunger, thirst, physical activity during the previous day and the current day, sleep pattern, as well as caffeine and cigarette consumption). Next, participants were prepared for EEG, which was collected from a BrainVision actiCAP system (Brain Products GmbH, Munich, Germany) labeled in accord with an extended international 10-20 system (Oostenveld \& Praamstra, 2001) and sampled at $250 \mathrm{~Hz}$. The EEG signal was referenced online to the left earlobe and a common ground was employed at the FPz electrode site. Electrode impedances were maintained below $25 \mathrm{k} \Omega$ throughout the study and a high-pass filter was set at $0.016 \mathrm{~Hz}$. A BrainAmp DC amplifier (Brain Products $\mathrm{GmbH}$ ) amplified and digitized the signal, which was recorded on a computer running BrainVision Recorder software (Brain Products GmbH). Participants were then seated $75 \mathrm{~cm}$ from the center of a $48 \mathrm{~cm}$ computer monitor and given instructions to facilitate EEG recording (e.g., avoid movement other than making required task responses).

Participants then completed the go/no-go task, which consisted of inhibit physical inactivity and inhibit physical activity conditions that used images from Kullmann et al. (2014). Stimuli depicted a model engaging in physical activity or inactivity, and the images were matched 
so that the only element that varied between them was the level of energy expenditure displayed by the model. As a control condition, a neutral go/no-go task used stimuli from the International Affective Picture System, half of which contained animals and the other half of which did not, and the images were matched based on past ratings of valence, arousal, and dominance (Lang et al., 2008). Condition order was randomized across participants. In the inhibit physical inactivity condition, participants were asked to respond as quickly as possible when an image depicting physical activity was presented on the screen ("go physical activity" trials) by pressing the response key on a keyboard (i.e., the space bar), and to not press the response key when an image depicting physical inactivity was presented on the screen ("no-go physical inactivity" trials). In the inhibit physical activity condition, the instructions were reversed - participants were asked to press the response key for an image depicting physical inactivity ("go physical inactivity" trials) and to not press the response key when an image depicting physical activity was presented on the screen ("no-go physical activity" trials). In the neutral condition, the images depicting physical activity and physical inactivity were replaced by the ones that included an animal or not. Half of the participants were asked to respond as quickly as possible when an image depicting an animal was presented on the screen ("go" trials) and to not press the response key when an image not depicting an animal was presented on the screen ("no-go" trials). For the other half of participants, the instructions were reversed. Each condition had 208 trials, $75 \%$ of which were go trials and $25 \%$ of which were nogo trials. Each trial began with an image presented for $500 \mathrm{~ms}$ followed by an inter-trial interval that randomly varied between 1200 and 1400 ms and during which a fixation cross was presented (Figure 1). The trial structure and go to no-go trial ratio in each condition was based on Carbine et al. (2017) ${ }^{1}$. Each condition began with eight practice trials (six go trials and two no-go trials), during which the researcher monitored the participants' performance to ensure they understood 
the task. After the practice trials preceding the first condition, the participant performed additional practice trials if they reported or exhibited confusion about the task (this was the case for one participant). After the practice trials preceding any condition, the experimenter reinforced instructions to the participant if they reported or exhibited confusion about the task.

After the final condition, participants completed a questionnaire assessing their personality and demographic characteristics, attitudes/intentions toward exercise, and usual physical activity behavior. Usual physical activity was measured with a short, self-administered version of the International Physical Activity Questionnaire (IPAQ; Craig et al., 2003) that asked participants to indicate how much moderate-to-vigorous physical activity they do during a usual week, as part of their everyday lives.

\section{EEG Data Processing}

EEG data processing was conducted with BrainVision Analzyer 2.2 software (Brain Products GmbH, Munich, Germany). Data were visually inspected, and malfunctioning electrodes were spherically interpolated, after which data were re-referenced to an average of the left and right ears. Next, data were prepared for independent component analysis (ICA) by applying an IIR $1-30 \mathrm{~Hz}$ bandpass filter with $4^{\text {th }}$ order roll-offs and $60 \mathrm{~Hz}$ notch filter. Then data exhibiting nonstereotypical artifact (e.g., non-ocular-related artifact) and data recorded between conditions was marked for exclusion from the ICA, which was run to identify components reflecting stereotypical artifacts (e.g., blinks and saccades). These components were then removed from the re-referenced, unfiltered data, which were then submitted to an IIR $0.1-30 \mathrm{~Hz}$ bandpass filter with $4^{\text {th }}$ order rolloffs and $60 \mathrm{~Hz}$ notch filter.

For the ERP (N2) analysis, data were segmented into epochs beginning $200 \mathrm{~ms}$ before and ending $1000 \mathrm{~ms}$ after image onset. Epochs were baseline corrected using the mean of the $200 \mathrm{~ms}$ 
period before image onset and then submitted to an automatic artifact rejection that removed epochs with $>50 \mu \mathrm{V} / \mathrm{ms}$ step, $>100 \mu \mathrm{V}$ change in a sliding $200 \mathrm{~ms}$ window, or $<0.5 \mu \mathrm{V}$ change in a sliding $200 \mathrm{~ms}$ window at a midline electrode $(\mathrm{Fz}, \mathrm{FCz}, \mathrm{Cz}$, or $\mathrm{Pz})$. This led to an average of $98.9 \%$ of 624 trials being retained per participant (minimum trials retained for a participant $=536$ [85.9\% of trials]). N2 was quantified as mean amplitude $200-300 \mathrm{~ms}$ after image onset, averaged across electrodes $\mathrm{FCz}$ and $\mathrm{Cz}$, based on Carbine et al. (2017), and extreme values $(<-50 \mu \mathrm{V}$ or $>$ $+50 \mu \mathrm{V})$ were deleted $(<0.01 \%$ trials were removed), based on past work from our lab (Lohse et al., 2020). We kept trials wherein participants made commission or omission errors, consistent with Carbine et al. (2017).

For the FM $\theta$ analysis, data were segmented into epochs beginning $500 \mathrm{~ms}$ before and ending $1000 \mathrm{~ms}$ after image onset. Epochs were then submitted to an automatic artifact rejection that removed epochs with $>50 \mu \mathrm{V} / \mathrm{ms}$ step, $>100 \mu \mathrm{V}$ change in a sliding $200 \mathrm{~ms}$ window, or $<0.5$ $\mu \mathrm{V}$ change in a sliding $200 \mathrm{~ms}$ window at a midline electrode (Fz, FCz, Cz, or Pz). This led to an average of $98.7 \%$ of 624 trials being retained per participant (minimum trials retained for a participant $=532[85.3 \%$ of trials $]$ ). Next, epochs were convolved with a 3 -cycle complex Morlet wavelet consisting of 20 frequencies between 1 and $40 \mathrm{~Hz}$ that increased logarithmically and was normalized with a z-transformation referenced to an interval $500-200 \mathrm{~ms}$ before image onset. For each participant, the wavelet between 3.206 and $6.969 \mathrm{~Hz}$ that exhibited the greatest peak power $300-700 \mathrm{~ms}$ after image onset at electrode $\mathrm{FCz}$ was identified and mean power between $300-$ $700 \mathrm{~ms}$ at electrode $\mathrm{FCz}$ was calculated, based on van de Vijver et al. (2018). Prior to statistical analyses, we visually inspected density plots and observed $99 \%$ of values fell between $-30 \mu \mathrm{V}^{2}$ and $+41 \mu \mathrm{V}^{2}$, but the full set of values ranged from -558 to $421 \mu \mathrm{V}^{2}$. To reduce the influence of these outlying scores, we excluded trials with values $<-50 \mu \mathrm{V}^{2}$ or $>+50 \mu \mathrm{V}^{2}$, as we did for the 
N2. We kept trials wherein participants made commission or omission errors, as we did for the N2.

\section{Statistical Analysis}

Behavioral (i.e., commission errors) and EEG (i.e., N2 and FM $\theta$ ) outcomes were tested using mixed effect models (MEM). MEM allow a correct estimation of parameters with multiple cross-random effects, such as in the present study in which participants are crossed with stimuli. MEM can also decrease the risk of type-I error (Boisgontier \& Cheval, 2016). All the analyses were conducted in $\mathrm{R}$ with the lme4 and lmerTest packages (Bates et al., 2014; Kuznetsova et al., 2015; R Core Team, 2017). To reduce convergence issues, each model was first optimized using the default BOBYQA optimizer (Powell, 2009), followed by the Nelder-Mead optimizer (Nelder \& Mead, 1965), the nlimb optimizer from the optimx package (Nash \& Varadhan, 2011), and then the L-BFGS-B optimizer (See Frossard \& Renaud, 2019, for similar procedure).

For commission errors, the model used has been described in detail elsewhere (Cheval, Daou, et al., 2020). In short, we used a logistic MEM to assess the association between the type of stimuli (physical inactivity vs. physical activity vs. neutral stimuli) and the commission errors, adjusting for the potential confounding influence of speed-accuracy trade-offs (i.e., median reaction time for the opposite stimuli in a given go/no go condition). Moreover, to investigate the influence of usual level of physical activity on commission errors, two-way interactions between the type of stimuli and the usual level of physical activity were added to the previous model. A significant interaction would indicate that the usual level of physical activity moderated the effect of the type of stimuli on commission errors.

For EEG outcomes (i.e., N2 and FM $\theta$ ), we used linear MEM that included the type of trials (i.e., go vs. no-go trials), the type of stimuli (physical inactivity vs. physical activity vs. neutral 
stimuli), as well as their interaction as fixed factors. The MEM specified both participants and stimuli as random factors, and included random effects for the type of stimuli and for the type of trials. Moreover, to investigate the influence of usual level of physical activity on the EEG outcomes, three-way interactions between the type of trials, the type of stimuli, and the usual level of physical activity were included in the models ${ }^{2}$. A moderating influence of the usual level of physical activity on the EEG outcomes would be evidenced by significant three-way or two-way interactions.

Estimates of the effect size were reported using the conditional pseudo $\mathrm{R}^{2}$ computed using the MuMin package (Barton, 2018). Regions of significance were estimated using the interactions package in R (Long, 2019). Statistical assumptions associated with MEM (i.e., normality of the residuals, linearity, multicollinearity, and undue influence) were checked and were met for all models.

\section{Results}

After the descriptive statistics, the results are reported in 3 sections: the first describes results of analyses on commission errors, the second describes results of analyses on $\mathrm{N} 2$, and the third describes results of analyses on FM $\theta$.

\section{Descriptive Statistics}

Table 1 shows participants' characteristics. The final sample included 50 participants (28 women; mean age $=21.6 \pm 2.2$ years). The usual level of moderate-to-vigorous physical activity was 559.5 min per week $( \pm 480.3 \mathrm{~min})$. Commission error rate was $29 \%$ for stimuli depicting physical inactivity, $22 \%$ for stimuli depicting physical activity, and $14 \%$ for neutral stimuli. Differences in $\mathrm{N} 2$ amplitudes between the no-go and go trials (no-go minus go trials) were $-.58 \mu \mathrm{V}$ for physical inactivity stimuli, $+.20 \mu \mathrm{V}$ for physical activity stimuli, and $-.94 \mu \mathrm{V}$ for neutral stimuli. Finally, 
differences in FM $\theta$ amplitudes between no-go and go trials (no-go minus go trials) were +2.33 $\mu \mathrm{V}^{2}$ for physical inactivity stimuli, $+2.37 \mu \mathrm{V}^{2}$ for physical activity stimuli, and $+3.06 \mu \mathrm{V}^{2}$ for neutral stimuli.

\section{Commission Errors}

The type of stimuli was associated with commission errors ( $p$ for global effect <.001). Compared with stimuli depicting physical activity, participants demonstrated higher commission errors for stimuli depicting physical inactivity (odds ratio $[\mathrm{OR}]=1.50,95 \%$ Confidence Interval $[95 \% \mathrm{CI}]=1.11$ to $2.01, p=.007)$, but lower commission errors for neutral stimuli $(\mathrm{OR}=.34$, $95 \% \mathrm{CI}=.24$ to $.50, p<.001)$. Slower median reaction times were associated with lower commission errors $(\mathrm{OR}=.72,95 \% \mathrm{CI}=.61$ to $.84, p<.001)$. However, median reaction time did not moderate the effect of the type of stimuli on commission errors ( $p s>318)$, suggesting that the effect of the type of stimuli was not related to a speed-accuracy trade-off (see Cheval et al., 2020 for further explanation). The variables under consideration explained $23.5 \%$ of the variance in the commission errors (Table 2; Figure 2). The associations between the type of stimuli and commission errors were not moderated by the usual level of physical activity $(p s>.110)$.

N2

Figure 3A shows grand average ERPs as a function of type of trial and stimuli as well as the N2 scalp distribution averaged across type of trial and stimuli. MEM results revealed a twoway interaction between the type of trials and the type of stimuli, suggesting that the effect of the type of trials on N2 significantly varied depending on the type of stimuli ( $p$ for global effect $=.001$ ). Simple interaction tests further revelated that the effect of the type of trials on N2 significantly differed between stimuli depicting physical activity compared with physical inactivity $(b=-.78 \mu \mathrm{V}$, $95 \% \mathrm{CI}=-1.38$ to $-.18 \mu \mathrm{V}, p=.011)$ or neutral $(\mathrm{b}=-1.11 \mu \mathrm{V}, 95 \% \mathrm{CI}=-1.71$ to $-.50 \mu \mathrm{V}, p<.001)$. No 
differences were observed between stimuli depicting physical inactivity and neutral stimuli $(b=.33$ $\mu \mathrm{V}, 95 \% \mathrm{CI}=-.27$ to $.93 \mu \mathrm{V}, p=.285)$. Simple effects showed a more negative N2 amplitude for nogo trials compared with go trials for both neutral stimuli $(b=-.91 \mu \mathrm{V}, 95 \% \mathrm{CI}=-1.42$ to $-.40 \mu \mathrm{V}$, $p<.001)$ and stimuli depicting physical inactivity $(\mathrm{b}=-.58 \mu \mathrm{V}, 95 \% \mathrm{CI}=-1.08$ to $-.08 \mu \mathrm{V}, p=.025)$. For stimuli depicting physical activity, results did not show any significant difference in N2 amplitude between no-go and go trials $(b=.20 \mu \mathrm{V}, 95 \% \mathrm{CI}=-0.31$ to $0.70 \mu \mathrm{V}, p=.445)$ (Table 3 , Figure 3B). The variables under consideration explained $11.5 \%$ of the variance in $\mathrm{N} 2$. The interactive effects between the type of trials and the type stimuli on N2 were not moderated by the usual level of physical activity $(p s>.330)$.

In sum, results revealed a typical no-go effect (i.e., more negative N2 amplitudes for no-go trials compared to go trials) for both neutral and physical inactivity stimuli, but no such effect was found for physical activity stimuli.

\section{FM}

Figure 4A shows grand average time-frequency plots as a function of type of trial and stimuli as well as FM $\theta$ scalp distribution averaged across type of trial and stimuli. Results revealed a two-way interaction between the type of trials and the type of stimuli, suggesting that the effect of the type of trials on FM $\theta$ significantly varied depending on the type of stimuli ( $p$ for global effect $=.030$ ). Simple interaction tests further revelated that the effect of the type of trials on FM $\theta$ was significantly less pronounced for physical activity $\left(b=.70 \mu \mathrm{V}^{2}, 95 \% \mathrm{CI}=.09\right.$ to $1.31 \mu \mathrm{V}^{2}$, $p=.026)$ and physical inactivity stimuli $\left(\mathrm{b}=-.74 \mu \mathrm{V}^{2}, 95 \% \mathrm{CI}=-1.35\right.$ to $\left.-.13 \mu \mathrm{V}^{2}, p=.018\right)$ compared with neutral stimuli. No significant differences were observed between stimuli depicting physical activity and physical inactivity $\left(\mathrm{b}=-0.04 \mu \mathrm{V}^{2}, 95 \% \mathrm{CI}=--0.64\right.$ to $\left.0.57 \mu \mathrm{V}^{2}, p=.900\right)$. Simple test effects further showed a significant effect of the type of trials, regardless the type of stimuli: greater 
FM $\theta$ was observed on the no-go trials compared to the go trials for physical inactivity stimuli $\left(b=2.32 \mu \mathrm{V}^{2}, 95 \% \mathrm{CI}=1.64\right.$ to $\left.3.00 \mu \mathrm{V}^{2}, p<.001\right)$, physical activity stimuli $\left(\mathrm{b}=2.36 \mu \mathrm{V}^{2}\right.$, $95 \% \mathrm{CI}=1.68$ to $\left.3.04 \mu \mathrm{V}^{2}, p<.001\right)$, and for neutral stimuli $\left(\mathrm{b}=3.06 \mu \mathrm{V}^{2}, 95 \% \mathrm{CI}=2.38\right.$ to $3.75 \mu \mathrm{V}^{2}$, $p<.001)$. The variables under consideration explained $5.1 \%$ of the variance in FM $\theta$.

In addition, results showed that the interactions between the type of trial and the type of stimuli was moderated by the usual level of physical activity (although $p$ for global effect $=.091$ ). Simple effect tests further revealed that usual level of physical activity significantly moderated the difference in the effect of the type of trials on FM $\theta$ between physical inactivity stimuli and neutral stimuli $\left(\mathrm{b}=-0.70 \mu \mathrm{V}^{2}, 95 \% \mathrm{CI}=-1.35\right.$ to $\left.-0.05 \mu \mathrm{V}^{2}, p=.034\right)$ as well as between physical inactivity stimuli and physical activity stimuli ( $\mathrm{b}=-0.82 \mu \mathrm{V}^{2}, 95 \% \mathrm{CI}=-1.47$ to $\left.-0.17 \mu \mathrm{V}^{2}, p=.014\right)$. The decomposition of these three-way interactions showed that the relationship between the typical nogo effect and usual physical activity was negative for physical inactivity stimuli but positive for physical activity and neutral stimuli. Specifically, for physical inactivity stimuli, results showed that the greater FM $\theta$ observed on the no-go trials compared to the go trials was less pronounced when the usual level of physical activity was higher. The region of significance of this no-go effect was no longer significant in highly active participants (+1.9 SD) (see supplemental material 1). By contrast, for physical activity stimuli, results showed that the greater FM $\theta$ observed on the no-go trials compared to the go trials was more pronounced when the usual level of physical activity was higher. However, the region of significance showed that the no-go effect was significant even in the least active individuals (see supplemental material 2).

In sum, results revealed that the no-go effect (i.e., more positive FM $\theta$ for no-go trials compared to go trials) for physical inactivity stimuli decreased as the usual level of physical activity increased. On the contrary, the no-go effect for physical activity stimuli showed the reverse 
pattern - increased as the usual level of physical activity increased. However, it should be noted that the usual level of physical activity did not significantly moderate the no-go effect for a given type of stimuli. The significant three-way interaction was observed because, for physical inactivity stimuli, the direction of the relationship between the no-go effect and usual level of physical activity was opposite from that of the two other types of stimuli.

\section{Discussion}

\section{Main Findings}

The present study drew on the data from Cheval, Daou, et al. (2020)'s study and used EEG to elucidate the neural mechanisms underlying the higher failure to withhold responses to physical inactivity stimuli. We found evidence that participants exhibited larger N2 for inhibiting responses (vs. responding) to physical inactivity stimuli, a result consistent with the typical no-go effect found in previous studies and that we reproduced here for the neutral stimuli (Folstein \& Van Petten, 2008). No significant effect on N2 was observed for physical activity stimuli. EEG results are consistent with behavioral results showing higher commission errors (i.e., failure to withhold the behavioral response) when participants inhibited their responses to physical inactivity stimuli, compared with physical activity stimuli. Hence, our study suggests that withholding responses to physical inactivity stimuli requires increased inhibitory control relative to withholding responses to physical activity stimuli (commission error and N2 results). Neither the N2 nor the commission error results were moderated by participants' usual level of physical activity. In the remainder of the discussion, we couch our results in light of previous studies and then we highlight the strengths and weaknesses of the current study.

\section{Comparison with Other Studies}


Results are consistent with a prior go/no-go study using EEG that revealed greater inhibitory control is demanded when avoiding attractive stimuli related to unhealthy eating behaviors (Carbine et al., 2017). However, whereas the prior study concerned energy consumption (e.g., low-calorie vs. high-calorie food), the present study focused on energy expenditure and showed results consistent with the assumption that cognitive resources are important for inhibiting responses to physical inactivity stimuli. Hence, our findings lend support to existing epidemiological and experimental studies (Cheval, Orsholits, et al., 2020; Cheval, Tipura, et al., 2018; Hall et al., 2008). For example, in Cheval, Tipura, et al. (2018)'s study, it was found that avoiding visual stimuli depicting sedentary behaviors was associated with larger N2 amplitudes. Furthermore, the current results align with recent perspectives (Buckley et al., 2014) and novel theories about physical inactivity and exercise (Brand \& Cheval, 2019; Brand \& Ekkekakis, 2018; Conroy \& Berry, 2017). In particular, results are largely in line with the TEMPA, from which the present study's hypotheses were drawn (Cheval \& Boisgontier, in press). Indeed, the TEMPA proposes that humans have an automatic attraction toward effort minimization, meaning extra cognitive resources are required to avoid it.

We found significant two-way interactions between the type of trials and the type of stimuli, revealing that the effect of the type of trials (i.e., the no-go effect) significantly differed between physical activity stimuli and neutral or physical inactivity stimuli, while no significant differences were observed between neutral and physical inactivity stimuli. Simple tests further revealed that the typical no-go effect observed in previous studies (i.e., higher N2 in no-go relative to go trials) (Folstein \& Van Petten, 2008) was observed for both neutral and physical inactivity stimuli, but not for physical activity stimuli. In other words, these results suggest that inhibiting responses to physical activity stimuli does not require as much inhibitory control as inhibiting 
responses to neutral or physical inactivity stimuli. These results contrasting physical activity and physical inactivity stimuli are consistent with previous literature that has suggested that people are naturally inclined to conserve energy and avoid unnecessary physical exertion (Brehm \& Self, 1989; Gendolla et al., 2012; Silvestrini \& Gendolla, 2013).

Crucially, it is important to note that we would have expected to observe a higher inhibitory control to withhold responses to physical inactivity stimuli relative to neutral stimuli, which was not the case. However, this absence of significant difference can be explained by the features of the neutral condition that we used. In particular, the neutral condition included images depicting an animal or a landscape rather than a simple symbol (e.g., a cross or a square), a strategy chosen to ensure that the complexity of the different conditions (i.e., the quantity of information to be processed) were approximately equivalent. Yet, although the valence and arousal were matched between the images that included an animal and the ones that included a landscape, they were not neutral (e.g., the average valence was $\sim 6.33$ out of 9 , indicating that they were perceived as pleasant). As such, the stimuli used in the neutral condition are likely to elicit affective responses and thereby may have biased participants' responses. Similarly, further analyses showed that a larger N2 was especially pronounced when participants were asked to inhibit their responses to images depicting a landscape compared with inhibiting responses to images depicting an animal. This unexpected pattern of results further reduces the confidence regarding the validity of the results of the neutral task. Future studies need to develop and validate a neutral condition to accurately determine the directionality of the effect - i.e., whether inhibiting responses to physical inactivity (or physical activity) stimuli are associated with higher vs. lower inhibitory control compared with inhibiting responses to neutral stimuli. Regardless of the directionality issue, by showing that inhibiting responses to physical inactivity stimuli requires significantly higher 
inhibitory control than inhibiting responses to physical activity stimuli, our findings are in line with TEMPA (Cheval \& Boisgontier, in press).

Further analyses on time-frequency data revealed that FM $\theta$ was higher for avoiding (vs. approaching) both physical inactivity and physical activity stimuli, and that participants' usual level of physical activity moderated these effects - greater FM $\theta$ for avoiding (vs. approaching) physical inactivity stimuli was stronger when usual level of physical activity was lower, while the FM $\theta$ effects for avoiding (vs. approaching) physical activity stimuli was stronger when usual level of physical activity was higher. This pattern of moderation was expected as we hypothesized that avoiding physical inactivity stimuli should require less inhibitory control in participants who manage to be physically active. However, this pattern should be interpreted in the light of our sample features - i.e., a highly active sample. Likewise, in such physically active participants, additional inhibitory control could be required to avoid physical activity stimuli, because these stimuli may have acquired, across time, a certain attractive or rewarding value (Cheval, Radel, et al., 2018; Crombie et al., 2018; Raichlen et al., 2012; Raichlen et al., 2013). In sum, these findings may suggest that highly active individuals need less inhibitory control to counteract the automatic attraction to effort minimization, but more inhibitory control to withhold responses to physical activity opportunities. Of note, this higher inhibitory control to withhold responses to physical activity stimuli has been already observed in participants with anorexia nervosa, a disorder characterized by physical hyperactivity (Giel et al., 2013).

\section{Strengths and Weaknesses}

The present study has a few limitations. First, the images used in the task showed a model in exercise clothing in an exercise context (e.g., on an exercise mat). This could have increased stimulus conflict when the model was physically inactive, which could have increased the N2 and 
FM $\theta$ to these stimuli (Cavanagh \& Frank, 2014). Future studies should avoid a potential confound with stimulus conflict by using "neutral" images (e.g., an avatar in an empty setting). Second, usual level of physical activity was indexed via self-report, which is prone to self-recall and desirability biases (Ward et al., 2005), thus limiting the ability to evaluate how participants' usual physical activity moderates the EEG effects observed. Finally, the present study involved individuals who were young and reported being highly physically active. These features limit the possibility to generalize the current results to other populations, such as older and/or less active individuals.

\section{Conclusion}

In conclusion, our results point out the neural mechanisms likely to underlie individuals' failure to withhold responses to physical inactivity stimuli by showing that inhibiting such stimuli requires inhibitory control compared with inhibiting responses to physical activity stimuli. These results may indicate that physical inactivity opportunities are automatically attractive and thereby difficult to avoid, as suggested by the theory of effort minimization in physical activity (TEMPA; Cheval \& Boisgontier, in press) and other theoretical perspectives (Brehm \& Self, 1989; Gendolla et al., 2012). By showing that the neural activity underlying the inhibition of responses to physical activity and inactivity stimuli significantly differed, our results shed light on the neuropsychological determinants of physical activity behaviors. 


\section{References}

Barton, K. (2018). MuMIn: Multi-model inference. R package version 1.42.1. https://CRAN.Rproject.org/package=MuMIn.

Bates, D., Mächler, M., Bolker, B., \& Walker, S. (2014). Fitting linear mixed-effects models using lme4. Journal of Statistical Software, 67(1), 1-48.

Bernacer, J., Martinez-Valbuena, I., Martinez, M., Pujol, N., Luis, E. O., Ramirez-Castillo, D., \& Pastor, M. A. (2019). An amygdala-cingulate network underpins changes in effort-based decision making after a fitness program. NeuroImage, 203, 116181.

Boisgontier, M. P., \& Cheval, B. (2016). The anova to mixed model transition. Neuroscience \& Biobehavioral Reviews, 68, 1004-1005.

Brand, R., \& Cheval, B. (2019). Theories to explain exercise motivation and physical inactivity: Ways of expanding our current theoretical perspective. Frontiers in Psychology, 10, 1147.

Brand, R., \& Ekkekakis, P. (2018). Affective-Reflective Theory of physical inactivity and exercise. German Journal of Exercise and Sport Research, 48(1), 48-58.

Brehm, J. W., \& Self, E. A. (1989). The intensity of motivation. Annual Review of Psychology, $40(1), 109-131$.

Buckley, J., Cohen, J. D., Kramer, A. F., McAuley, E., \& Mullen, S. P. (2014). Cognitive control in the self-regulation of physical activity and sedentary behavior. Frontiers in Human Neuroscience, 8, 747.

Bull, F. C., Al-Ansari, S. S., Biddle, S., Borodulin, K., Buman, M. P., Cardon, G., Carty, C., Chaput, J.-P., Chastin, S., \& Chou, R. (2020). World Health Organization 2020 guidelines on physical activity and sedentary behaviour. British Journal of Sports Medicine, 54(24), 1451-1462. 
Carbine, K. A., Christensen, E., LeCheminant, J. D., Bailey, B. W., Tucker, L. A., \& Larson, M. J. (2017). Testing food-related inhibitory control to high-and low-calorie food stimuli: Electrophysiological responses to high-calorie food stimuli predict calorie and carbohydrate intake. Psychophysiology, 54(7), 982-997.

Cavanagh, J. F., \& Frank, M. J. (2014). Frontal theta as a mechanism for cognitive control. Trends in Cognitive Sciences, 18(8), 414-421.

CFLRI. (2018). Canadian Fitness and Lifestyle Research Institute. Physical activity monitor: facts and figures. http://www.cflri.ca/sites/default/files/node/95/files/PAM2008FactsFigures_Bulletin14_Int ention to be activeEN.pdf.

Cheval, B., \& Boisgontier, M. P. (in press). The theory of effort minimization in physical activity (TEMPA). Exercise and Sport Sciences Reviews.

Cheval, B., Daou, M., Cabral, D. A. R., Bacelar, M., Parma, J. O., Forestier, C., Orsholits, D., Sander, D., Boisgontier, M. P., \& Miller, M. W. (2020). Higher inhibitory control is required to escape the innate attraction to effort minimization. Psychology of Sport and Exercise, 51, 101781.

Cheval, B., Miller, M. W., Orsholits, D., Berry, T., Sander, D., \& Boisgontier, M. P. (2020). Physically active individuals look for more: an eye-tracking study of attentional bias. Psychophysiology, 57(6), e13582.

Cheval, B., Orsholits, D., Sieber, S., Courvoisier, D. C., Cullati, S., \& Boisgontier, M. P. (2020). Relationship between decline in cognitive resources and physical activity. Health Psychology, 39(6), 519-528. 
Cheval, B., Radel, R., Neva, J. L., Boyd, L. A., Swinnen, S. P., Sander, D., \& Boisgontier, M. P. (2018). Behavioral and neural evidence of the rewarding value of exercise behaviors: a systematic review. Sports Medicine, 48(6), 1389-1404.

Cheval, B., Rebar, A. L., Miller, M. M., Sieber, S., Orsholits, D., Baranyi, G., Courvoisier, D. C., Cullati, S., Sander, D., \& Boisgontier, M. P. (2019). Cognitive resources moderate the adverse impact of poor neighborhood conditions on physical activity. Preventive Medicine, $126,105741$.

Cheval, B., Sarrazin, P., Boisgontier, M. P., \& Radel, R. (2017). Temptations toward behaviors minimizing energetic costs (BMEC) automatically activate physical activity goals in successful exercisers. Psychology of Sport and Exercise, 30, 110-117.

Cheval, B., Sarrazin, P., Isoard-Gautheur, S., Radel, R., \& Friese, M. (2015). Reflective and impulsive processes explain (in)effectiveness of messages promoting physical activity: a randomized controlled trial. Health Psychology, 34(1), 10-19.

Cheval, B., Sarrazin, P., \& Pelletier, L. (2014). Impulsive approach tendencies towards physical activity and sedentary behaviors, but not reflective intentions, prospectively predict nonexercise activity thermogenesis. Plos One, 9(12), e115238.

Cheval, B., Tipura, E., Burra, N., Frossard, J., Chanal, J., Orsholits, D., Radel, R., \& Boisgontier, M. P. (2018). Avoiding sedentary behaviors requires more cortical resources than avoiding physical activity: an EEG study. Neuropsychologia, 119, 68-80.

Conroy, D. E., \& Berry, T. R. (2017). Automatic affective evaluations of physical activity. Exercise and Sport Sciences Reviews, 45(4), 230-237. 
Conroy, D. E., Hyde, A. L., Doerksen, S. E., \& Ribeiro, N. F. (2010). Implicit attitudes and explicit motivation prospectively predict physical activity. Annals of Behavioral Medicine, 39(2), 112-118.

Craig, C. L., Marshall, A. L., Sjostrom, M., Bauman, A. E., Booth, M. L., Ainsworth, B. E., Pratt, M., Ekelund, U., Yngve, A., Sallis, J. F., \& Oja, P. (2003). International physical activity questionnaire: 12-country reliability and validity. Medicine and Science in Sports and Exercise, 35(8), 1381-1395. $<$ Go to ISI $>: / / 000184527600020$

Crombie, K. M., Brellenthin, A. G., Hillard, C. J., \& Koltyn, K. F. (2018). Endocannabinoid and opioid system interactions in exercise-induced hypoalgesia. Pain Medicine, 19(1), 118123.

Daly, M., McMinn, D., \& Allan, J. L. (2015). A bidirectional relationship between physical activity and executive function in older adults. Frontiers in Human Neuroscience, 8, 1044.

Ding, D., Lawson, K. D., Kolbe-Alexander, T. L., Finkelstein, E. A., Katzmarzyk, P. T., van Mechelen, W., Pratt, M., \& Committee, L. P. A. S. E. (2016). The economic burden of physical inactivity: a global analysis of major non-communicable diseases. The Lancet, 388(10051), 1311-1324.

Faul, F., Erdfelder, E., Lang, A.-G., \& Buchner, A. (2007). G* Power 3: A flexible statistical power analysis program for the social, behavioral, and biomedical sciences. Behavior Research Methods, 39(2), 175-191.

Folstein, J. R., \& Van Petten, C. (2008). Influence of cognitive control and mismatch on the N2 component of the ERP: a review. Psychophysiology, 45(1), 152-170.

Frossard, J., \& Renaud, O. (2019). The correlation structure of mixed effects models with crossed random effects in controlled experiments. arXiv. 
Gendolla, G. H., Wright, R. A., \& Richter, M. (2012). Effort intensity: Some insights from the cardiovascular system. The Oxford handbook of human motivation, 420-438.

Giel, K. E., Kullmann, S., Preiß1, H., Bischoff, S. C., Thiel, A., Schmidt, U., Zipfel, S., \& Teufel, M. (2013). Understanding the reward system functioning in anorexia nervosa: crucial role of physical activity. Biological Psychology, 94(3), 575-581.

Guthold, R., Stevens, G. A., Riley, L. M., \& Bull, F. C. (2018). Worldwide trends in insufficient physical activity from 2001 to 2016: a pooled analysis of 358 population-based surveys with 1. 9 million participants. The Lancet Global Health, 6(10), e1077-1086.

Hall, P. A., Fong, G. T., Epp, L. J., \& Elias, L. J. (2008). Executive function moderates the intention-behavior link for physical activity and dietary behavior. Psychology \& Health, 23(3), 309-326.

Klein-Flügge, M. C., Kennerley, S. W., Friston, K., \& Bestmann, S. (2016). Neural signatures of value comparison in human cingulate cortex during decisions requiring an effort-reward trade-off. Journal of Neuroscience, 36(39), 10002-10015.

Kullmann, S., Giel, K. E., Hu, X., Bischoff, S. C., Teufel, M., Thiel, A., Zipfel, S., \& Preissl, H. (2014). Impaired inhibitory control in anorexia nervosa elicited by physical activity stimuli. Social Cognitive and Affective Neuroscience, 9(7), 917-923.

Kuznetsova, A., Brockhoff, P. B., \& Christensen, R. H. B. (2015). lmerTest Package: tests in linear mixed effects models. Journal of Statistical Software, 82(13), 1-26.

Lang, P., Bradley, M., \& Cuthbert, B. (2008). International affective picture system (IAPS): affective ratings of pictures and instruction manual. University of Florida, Gainesville.

Lee, H. H., Emerson, J., \& Williams, D. (2016). The exercise-affect-adherence pathway: an evolutionary perspective. Frontiers in Psychology, 7, 1285. 
Lee, I.-M., Shiroma, E. J., Lobelo, F., Puska, P., Blair, S. N., Katzmarzyk, P. T., \& Group, L. P. A. S. W. (2012). Effect of physical inactivity on major non-communicable diseases worldwide: an analysis of burden of disease and life expectancy. The Lancet, 380(9838), 219-229.

Lieberman, D. E. (2015). Is exercise really medicine? An evolutionary perspective. Current Sports Medicine Reports, 14(4), 313-319.

Lindwall, M., Cimino, C. R., Gibbons, L. E., Mitchell, M. B., Benitez, A., Brown, C. L., Kennison, R. F., Shirk, S. D., Atri, A., \& Robitaille, A. (2012). Dynamic associations of change in physical activity and change in cognitive function: coordinated analyses of four longitudinal studies. Journal of Aging Research, 2012, 493598.

Lohse, K., Miller, M., Daou, M., Valerius, W., \& Jones, M. (2020). Dissociating the contributions of reward-prediction errors to trial-level adaptation and long-term learning. Biological Psychology, 149, 107775.

Long, J. (2019). interactions: Comprehensive, User-Friendly Toolkit for Probing Interactions. R package version 1.1.0, https://cran.r-project.org/package=interactions.

Martin, S. B., Morrow, J. R., Jackson, A. W., \& Dunn, A. L. (2000). Variables related to meeting the CDC/ACSM physical activity guidelines. Medicine and Science in Sports and Exercise, 32(12), 2087-2092.

Moffitt, R. L., Kemps, E., Hannan, T. E., Neumann, D. L., Stopar, S. P., \& Anderson, C. J. (2019). Implicit approach biases for physically active lifestyle cues. International Journal of Sport and Exercise Psychology, 1-17.

Nash, J. C., \& Varadhan, R. (2011). Unifying optimization algorithms to aid software system users: optimx for R. Journal of Statistical Software, 43(9), 1-14. 
Nelder, J. A., \& Mead, R. (1965). A simplex method for function minimization. The Computer Journal, 7(4), 308-313.

Oostenveld, R., \& Praamstra, P. (2001). The five percent electrode system for high-resolution EEG and ERP measurements. Clinical neurophysiology, 112(4), 713-719.

Powell, M. J. (2009). The BOBYQA algorithm for bound constrained optimization without derivatives. Cambridge NA Report NA2009/06, University of Cambridge, Cambridge, 2646.

R Core Team. (2017). R: A language and environment for statistical computing. https://www.Rproject.org/

Raichlen, D. A., Foster, A. D., Gerdeman, G. L., Seillier, A., \& Giuffrida, A. (2012). Wired to run: exercise-induced endocannabinoid signaling in humans and cursorial mammals with implications for the 'runner's high'. Journal of Experimental Biology, 215(8), 1331-1336.

Raichlen, D. A., Foster, A. D., Seillier, A., Giuffrida, A., \& Gerdeman, G. L. (2013). Exerciseinduced endocannabinoid signaling is modulated by intensity. European Journal of Applied Physiology, 113(4), 869-875.

Rebar, A. L., Ram, N., \& Conroy, D. E. (2015). Using the EZ-diffusion model to score a singlecategory implicit association test of physical activity. Psychology of Sport and Exercise, $16(3), 96-105$.

Rouse, P. C., Ntoumanis, N., \& Duda, J. L. (2013). Effects of motivation and depletion on the ability to resist the temptation to avoid physical activity. International Journal of Sport and Exercise Psychology, 11(1), 39-56. 
Sabia, S., Dugravot, A., Dartigues, J.-F., Abell, J., Elbaz, A., Kivimäki, M., \& Singh-Manoux, A. (2017). Physical activity, cognitive decline, and risk of dementia: 28 year follow-up of Whitehall II cohort study. BMJ, 357, j2709.

Silvestrini, N., \& Gendolla, G. H. (2013). Automatic effort mobilization and the principle of resource conservation: One can only prime the possible and justified. Journal of Personality and Social Psychology, 104(5), 803-816.

Snowden, M., Steinman, L., Mochan, K., Grodstein, F., Prohaska, T. R., Thurman, D. J., Brown, D. R., Laditka, J. N., Soares, J., \& Zweiback, D. J. (2011). Effect of exercise on cognitive performance in community-dwelling older adults: review of intervention trials and recommendations for public health practice and research. Journal of the American Geriatrics Society, 59(4), 704-716.

Speakman, J. R. (2019). An evolutionary perspective on sedentary behavior. Bioessays, 42(1), e1900156.

van de Vijver, I., van Schie, H. T., Veling, H., van Dooren, R., \& Holland, R. W. (2018). Go/nogo training affects frontal midline theta and mu oscillations to passively observed food stimuli. Neuropsychologia, 119, 280-291.

Ward, D. S., Evenson, K. R., Vaughn, A., Rodgers, A. B., \& Troiano, R. P. (2005). Accelerometer use in physical activity: best practices and research recommendations. Medicine and Science in Sports and Exercise, 37(11 Suppl), S582-588.

WHO. (2020). WHO guidelines on physical activity and sedentary behaviour. https://www.who.int/publications/i/item/9789240015128. Accessed December 2020. 
Young, J., Angevaren, M., Rusted, J., \& Tabet, N. (2015). Aerobic exercise to improve cognitive function in older people without known cognitive impairment. The Cochrane database of systematic reviews, 4, CD005381. 


\section{Footnotes}

${ }^{1}$ We wanted to present all 26 of Kullmann et al.'s images equally in go and no-go conditions, so we increased the number of trials from 200 to 208 , which is a factor of 26 , and decreased the percentage of no-go trials from $30 \%$ to $25 \%$, to allow each image to be presented 2 times in a nogo condition and 6 times in a go condition. These slight modifications worked to our advantage by increasing our number trials and, thus, signal-to-noise ratio, and decreasing the likelihood of a trial being a no-go trial, thereby possibly increasing demands on inhibitory control.

${ }^{2}$ In the pre-registration, we indicated that we would use N2/FM $\theta$ as independent variables to explain usual physical activity (dependent variable). We changed this strategy to leverage the benefits of MEM (i.e., treating both participants and stimuli as random, avoiding having to average over observations, returning acceptable type I error rate), as well as to be consistent with the procedure adopted in Cheval et al. (2020). Specifically, we used usual physical activity as a potential moderating variable of the effect of type of trial and stimuli on N2/FM $\theta$. 


\section{Declarations}

\section{Funding}

B.C. is supported by an Ambizione grant (PZ00P1_180040) from the Swiss National Science Foundation (SNSF).

\section{Competing interests}

The authors declare no conflict of interests.

\section{Ethical approval}

This study was approved by the Auburn University Institutional Review Board (\#19-173 EP 1905).

\section{Consent to participate}

All the participants agreed to participate and signed a written informed consent

\section{Consent for publication}

All the authors listed in the by-line have agreed to the by-line order and to the submission of the manuscript in this form.

\section{Availability of data and material}

The dataset is available at https://zenodo.org/record/4421252\#.X W4SC1zg3g

\section{Code availability}

The code is available at https://zenodo.org/record/4421252\#.X W4SC1zg3g

\section{Contributors}

B.C. and M.W.M. designed the study protocol and the analyses. M.D. and D.A.R.C. collected the data. B.C., D.A.R.C., M.F.B.B., and J.O.P. analyzed the data. B.C. and M.W.M drafted the manuscript. All authors critically appraised and approved the final version of the manuscript. 


\section{Table 1}

Descriptive statistics

\begin{tabular}{|c|c|c|}
\hline $\mathrm{N}=50$ & Mean & SD \\
\hline Age (years) & 21.6 & 2.2 \\
\hline Gender (number; \% women) & 28 & $56.0 \%$ \\
\hline Intention to be active (Likert scale; $1-10$ ) & 8.5 & 1.7 \\
\hline Usual level of MVPA (minutes) & 559.5 & 480.3 \\
\hline \multicolumn{3}{|c|}{ Number commission errors (\% of errors; SD) } \\
\hline neutral stimuli & $16 \%$ & $20 \%$ \\
\hline physical activity stimuli & $24 \%$ & $16 \%$ \\
\hline physical inactivity stimuli & $31 \%$ & $18 \%$ \\
\hline \multicolumn{3}{|l|}{ N2 ERP $(\mu V)$} \\
\hline \multicolumn{3}{|l|}{ Go trials } \\
\hline neutral stimuli & -5.17 & 3.70 \\
\hline physical activity stimuli & -4.14 & 3.27 \\
\hline physical inactivity stimuli & -3.76 & 3.16 \\
\hline \multicolumn{3}{|l|}{ No-go trials } \\
\hline neutral stimuli & -6.11 & 4.19 \\
\hline physical activity stimuli & -3.94 & 3.67 \\
\hline physical inactivity stimuli & -4.34 & 3.14 \\
\hline \multicolumn{3}{|c|}{ Relative difference between No-go and go trials (No-go minus Go trials) } \\
\hline neutral stimuli & -0.94 & \\
\hline physical activity stimuli & 0.20 & \\
\hline physical inactivity stimuli & -0.58 & \\
\hline \multicolumn{3}{|l|}{ FMO $\left(\mu \mathrm{V}^{2}\right)$} \\
\hline \multicolumn{3}{|l|}{ Go trials } \\
\hline neutral stimuli & 4.55 & 1.46 \\
\hline physical activity stimuli & 4.78 & 1.70 \\
\hline physical inactivity stimuli & 4.86 & 1.76 \\
\hline \multicolumn{3}{|l|}{ No-go trials } \\
\hline neutral stimuli & 7.61 & 3.79 \\
\hline physical activity stimuli & 7.15 & 3.17 \\
\hline physical inactivity stimuli & 7.19 & 3.12 \\
\hline \multicolumn{3}{|c|}{ Relative difference between No-go and go trials (No-go minus Go trials) } \\
\hline neutral stimuli & 3.06 & \\
\hline physical activity stimuli & 2.37 & \\
\hline physical inactivity stimuli & 2.33 & \\
\hline
\end{tabular}

Notes. $\mathrm{SD}=$ standard deviation; $\mathrm{ms}=$ milliseconds; $\mathrm{MVPA}=$ moderate-to-vigorous physical activity 
Table 2

Results of the mixed models predicting commission errors

\begin{tabular}{|c|c|c|c|c|}
\hline \multirow[t]{2}{*}{ Commission error } & \multicolumn{2}{|c|}{$\begin{array}{c}\text { Without MVPA } \\
(n=49)\end{array}$} & \multicolumn{2}{|c|}{$\begin{array}{c}\text { With MVPA** } \\
(n=42)\end{array}$} \\
\hline & $\mathrm{OR}(\mathrm{CI})$ & $p$ & $\mathrm{OR}(\mathrm{CI})$ & $p$ \\
\hline \multicolumn{5}{|l|}{ Fixed Effects } \\
\hline Intercept & $0.26(0.20 ; 0.34)$ & $<.001$ & $0.39(0.30 ; 0.51)$ & $<.001$ \\
\hline \multicolumn{5}{|l|}{ Stimuli (ref. physical activity stimuli) } \\
\hline Physical inactivity & $1.50(1.11 ; 2.01)$ & .007 & $1.56(1.15 ; 2.13)$ & .004 \\
\hline Neutral & $0.34(0.24 ; 0.50)$ & $<.001$ & $0.36(0.24 ; 0.53)$ & $<.001$ \\
\hline \multicolumn{5}{|l|}{ Median reaction time } \\
\hline Median reaction time & $0.72(0.61 ; 0.84)$ & $<.001$ & $0.78(0.65 ; 0.92)$ & .003 \\
\hline Physical inactivity stimuli $x$ median reaction & $1.10(0.91 ; 1.34)$ & .318 & $1.06(0.87 ; 1.30)$ & .553 \\
\hline \multicolumn{5}{|l|}{ time } \\
\hline Neutral stimuli x median reaction time & $1.03(0.73 ; 1.47)$ & .855 & $0.93(0.65 ; 1.33)$ & .690 \\
\hline \multicolumn{5}{|l|}{ Usual level of physical activity } \\
\hline \multicolumn{3}{|c|}{ Usual level of physical activity } & $1.02(0.84 ; 1.24)$ & .816 \\
\hline Usual level of physical activity x Physical inac & tivity stimuli & & $1.00(0.83 ; 1.21)$ & .960 \\
\hline Usual level of physical activity x Neutral stim & $1 \mathrm{i}$ & & $1.21(0.96 ; 1.54)$ & .110 \\
\hline$p$-value for global effect & $<.001$ & & & \\
\hline \multicolumn{5}{|l|}{ Random Effects } \\
\hline \multicolumn{5}{|l|}{ Participants } \\
\hline Intercept & \multicolumn{2}{|l|}{0.308} & \multicolumn{2}{|l|}{0.208} \\
\hline Stimuli physical inactivity & \multicolumn{2}{|l|}{0.119} & \multicolumn{2}{|l|}{0.095} \\
\hline Stimuli neutral & \multicolumn{2}{|l|}{0.118} & \multicolumn{2}{|l|}{0.280} \\
\hline Corr. (Intercept, stimuli physical inactivity) & \multicolumn{2}{|l|}{0.04} & \multicolumn{2}{|l|}{0.34} \\
\hline Corr. (Intercept, stimuli neutral) & \multicolumn{2}{|l|}{0.09} & \multicolumn{2}{|l|}{-0.33} \\
\hline $\begin{array}{l}\text { Corr. (Stimuli physical inactivity; stimuli } \\
\text { neutral) }\end{array}$ & \multicolumn{2}{|l|}{0.82} & \multicolumn{2}{|l|}{0.72} \\
\hline \multicolumn{5}{|l|}{ Stimuli } \\
\hline Intercept & \multicolumn{2}{|l|}{0.274} & \multicolumn{2}{|l|}{0.278} \\
\hline \multicolumn{5}{|l|}{ Residual } \\
\hline & \multicolumn{2}{|l|}{.235} & \multicolumn{2}{|l|}{.213} \\
\hline
\end{tabular}


Table 3

Results of the mixed models predicting N2 ERP

\begin{tabular}{|c|c|c|c|c|}
\hline \multirow[t]{2}{*}{$\mathbf{N 2}$} & \multicolumn{2}{|c|}{$\begin{array}{l}\text { Without MVPA } \\
\quad(n=50)\end{array}$} & \multicolumn{2}{|l|}{$\begin{array}{l}\text { With MVPA* } \\
\quad(n=43)\end{array}$} \\
\hline & $\mathrm{b}(\mathrm{CI})$ & $p$ & $\mathrm{~b}(\mathrm{CI})$ & $p$ \\
\hline \multicolumn{5}{|l|}{ Fixed Effects } \\
\hline Intercept & $-4.30(-5.25 ;-3.35)$ & $<.001$ & $-4.10(-5.05 ;-3.13)$ & $<.001$ \\
\hline \multicolumn{5}{|l|}{ Type of trials (ref. Go trials) } \\
\hline No-go trials & $.20(-.31 ; .70)$ & .445 & $.24(-.30 ; .78)$ & .387 \\
\hline \multicolumn{5}{|l|}{ Stimuli (ref. physical activity stimuli) } \\
\hline Physical inactivity & $.41(-.18 ; .99)$ & .176 & $.40(-.22 ; 1.01)$ & .207 \\
\hline Neutral & $-.76(-1.56 ; .03)$ & .063 & $-.76(-1.64 ; .12)$ & .094 \\
\hline \multicolumn{5}{|c|}{ Type of trials (ref. Go trials) x Stimuli (ref. physical activity stimuli) } \\
\hline Physical inactivity stimuli x No-go trials & $-.78(-1.37 ;-.18)$ & .011 & $-.73(-1.36 ;-.09)$ & .026 \\
\hline Neutral stimuli x No-go trials & $-1.11(-1.70 ;-.50)$ & $<.001$ & $-1.16(-1.81 ;-.52)$ & $<.001$ \\
\hline \multicolumn{5}{|l|}{ Usual level of physical activity } \\
\hline Usual level of physical activity & & & $-.69(-1.61 ; .22)$ & .144 \\
\hline Usual level of physical activity $\mathrm{x}$ Physical ina & ctivity stimuli & & $-.11(-.58 ; .35)$ & .637 \\
\hline Usual level of physical activity $\mathrm{x}$ Neutral stin & & & $.03(-.76 ; .83)$ & .933 \\
\hline Usual level of physical activity $\mathrm{x}$ No go trials & & & $-.46(-.08 ; 1.01)$ & .097 \\
\hline Usual level of physical activity $\mathrm{x}$ Physical ina & ctivity stimuli $\mathrm{x}$ No g & trials & $-.32(-.96 ; .32)$ & .330 \\
\hline Usual level of physical activity $\mathrm{x}$ Physical Ne & atral stimuli x No-go & rials & $-.28(-.93 ; .36)$ & .389 \\
\hline$p$-value for global effect & $<.001$ & & & \\
\hline \multicolumn{5}{|l|}{ Random Effects } \\
\hline \multicolumn{5}{|l|}{ Participants } \\
\hline Intercept & 9.920 & & 8.788 & \\
\hline Stimuli physical inactivity & 1.155 & & 1.297 & \\
\hline Stimuli neutral & 5.240 & & 5.971 & \\
\hline \multicolumn{5}{|l|}{ Type of trials } \\
\hline Corr. (Intercept, stimuli physical inactivity) & -.36 & & -.39 & \\
\hline Corr. (Intercept, stimuli neutral) & -.19 & & -.28 & \\
\hline \multicolumn{5}{|l|}{ neutral) } \\
\hline Corr. (Intercept, type of trials) & -.06 & & -.12 & \\
\hline Corr. (Stimuli neutral, type of trials) & 0 & & -.02 & \\
\hline $\begin{array}{l}\text { Corr. (Stimuli physical inactivity, type of } \\
\text { trials) }\end{array}$ & -.19 & & -.19 & \\
\hline \multicolumn{5}{|l|}{ Stimuli } \\
\hline Intercept & .864 & & .840 & \\
\hline Residual & 89.53 & & 88.05 & \\
\hline $\mathrm{R}^{2}$ & .116 & & .108 & \\
\hline
\end{tabular}

Notes. $\mathrm{CI}=$ confidence interval at $95 \%$; MVPA=moderate-to-vigorous physical activity; $*$ With MVPA the final sample size is $n=42$, because 7 participants did not report their usual physical activity level. 
Table 4

Results of the mixed models predicting Frontal Midline Theta (FMO)

\begin{tabular}{|c|c|c|c|c|}
\hline \multirow[t]{2}{*}{ Frontal Midline Theta (FMO) } & \multicolumn{2}{|c|}{$\begin{array}{l}\text { Without MVPA } \\
(n=50)\end{array}$} & \multicolumn{2}{|c|}{$\begin{array}{l}\text { With MVPA } \\
(n=43)\end{array}$} \\
\hline & $\mathrm{b}(\mathrm{CI})$ & $p$ & $\mathrm{~b}(\mathrm{CI})$ & $p$ \\
\hline \multicolumn{5}{|l|}{ Fixed Effects } \\
\hline Intercept & $4.77(4.29 ; 5.24)$ & $<.001$ & $4.82(4.31 ; 5.32)$ & $<.001$ \\
\hline \multicolumn{5}{|l|}{ Type of trials (ref. Go trials) } \\
\hline No-go trials & $2.36(1.68 ; 3.04)$ & $<.001$ & $2.12(1.43 ; 2.83)$ & $<.001$ \\
\hline \multicolumn{5}{|l|}{ Stimuli (ref. physical activity stimuli) } \\
\hline Physical inactivity & $.10(-0.31 ; .50)$ & .645 & $-.13(-.56 ; .31)$ & .574 \\
\hline Neutral & $-.22(-1.56 ; .03)$ & .396 & $-.27(-.80 ; .25)$ & .310 \\
\hline \multicolumn{5}{|c|}{ Type of trials (ref. Go trials) x Stimuli (ref. physical activity stimuli) } \\
\hline Physical inactivity stimuli x No-go trials & $-.04(-.644 ; .57)$ & .900 & $-.41(-.24 ; 1.05)$ & .218 \\
\hline Neutral stimuli x No-go trials & $.70(0.09 ; 1.31)$ & .026 & $.71(.05 ; 1.36)$ & .034 \\
\hline \multicolumn{5}{|l|}{ Usual level of physical activity } \\
\hline Usual level of physical activity & & & $-.15(-.64 ; .35)$ & .563 \\
\hline Usual level of physical activity x Physical & ctivity stimuli & & $-.06(-.47 ; .35)$ & .781 \\
\hline Usual level of physical activity $x$ Neutral s & uli & & $.05(-.46 ; .55)$ & .863 \\
\hline Usual level of physical activity x No-go tr & & & $.15(-.55 ; .85)$ & 678 \\
\hline Usual level of physical activity x Physical & ctivity stimuli $\mathrm{x}$ N & o trials & $-.70(-1.35 ;-.05)$ & .034 \\
\hline Usual level of physical activity x Physical & utral stimuli x No- & trials & $.16(-.54 ; .77)$ & .728 \\
\hline$p$-value for global effect & $<.001$ & & $=.091$ & \\
\hline \multicolumn{5}{|l|}{ Random Effects } \\
\hline \multicolumn{5}{|l|}{ Participants } \\
\hline Intercept & 2.214 & & 2.172 & \\
\hline Stimuli physical inactivity & .721 & & .766 & \\
\hline Stimuli neutral & 1.814 & & 1.734 & \\
\hline \multicolumn{5}{|l|}{ Type of trials } \\
\hline \multicolumn{5}{|l|}{ inactivity) } \\
\hline Corr. (Intercept, stimuli neutral) & -.54 & & -.49 & \\
\hline Corr. (Stimuli physical inactivity; stimuli & .33 & & .45 & \\
\hline \multicolumn{5}{|l|}{ neutral) } \\
\hline Corr. (Intercept, type of trials) & .24 & & .24 & \\
\hline Corr. (Stimuli neutral, type of trials) & .01 & & .02 & \\
\hline $\begin{array}{l}\text { Corr. (Stimuli physical inactivity, type of } \\
\text { trials) }\end{array}$ & .14 & & .11 & \\
\hline \multicolumn{5}{|l|}{ Stimuli } \\
\hline Intercept & .072 & & .085 & \\
\hline Residual & 86.68 & & 85.27 & \\
\hline $\mathrm{R}^{2}$ & .051 & & .049 & \\
\hline
\end{tabular}


Notes. $\mathrm{CI}=$ confidence interval at $95 \%$; MVPA=moderate-to-vigorous physical activity; $*$ With MVPA the final sample size is $n=42$, because 7 participants did not report their usual physical activity level. 


\section{Figure 1}

\section{Go/no-go tasks}

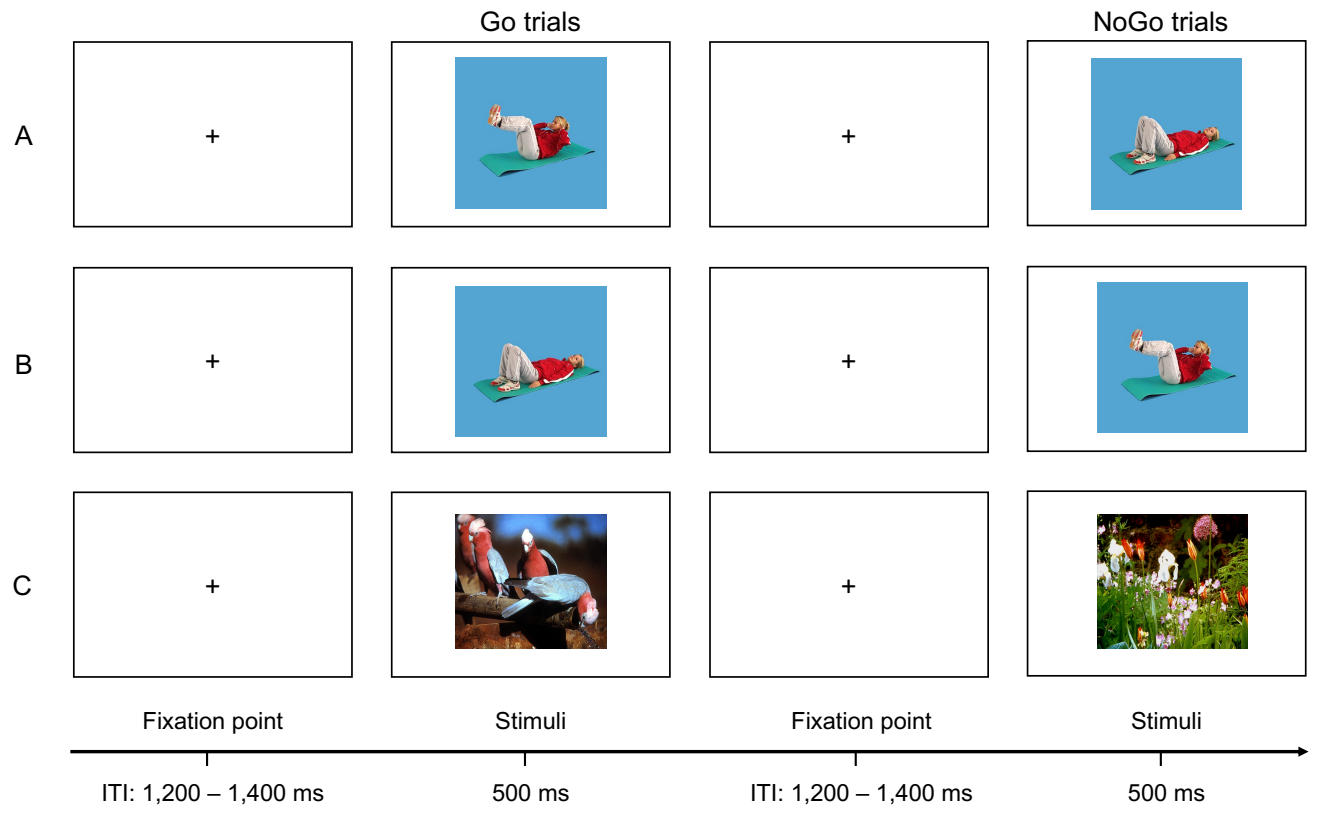

Note. The experiment consisted of three go/no-go conditions of 208 trials (go trials, $75 \%$ occurrence; no-go trials, $25 \%$ occurrence). A. Avoid physical inactivity condition. In this condition, participants were instructed to respond to physical activity images and to not respond to physical inactivity images. B. Avoid physical activity condition. In this condition, participants were instructed to respond to physical inactivity images and to not respond to physical activity images. C. Neutral condition. In this third condition, the stimuli depicting physical activity and physical inactivity were replaced by stimuli including an animal versus not including an animal (neutral condition). Participants were either asked to respond to images depicting an animal and to not respond to images not depicting an animal (this condition is depicted in the figure), or to do the reverse. The order of conditions was randomized for each participant. The random intertrial interval (ITI) varied between 1,200 and 1,400 ms. Stimuli were presented for $500 \mathrm{~ms}$. 


\section{Figure 2}

Go/no-go behavioral outcome. Commission error. The odds ratio of a failure of inhibition in the no-go trials to stimuli depicting physical activity, neutral, and physical inactivity.

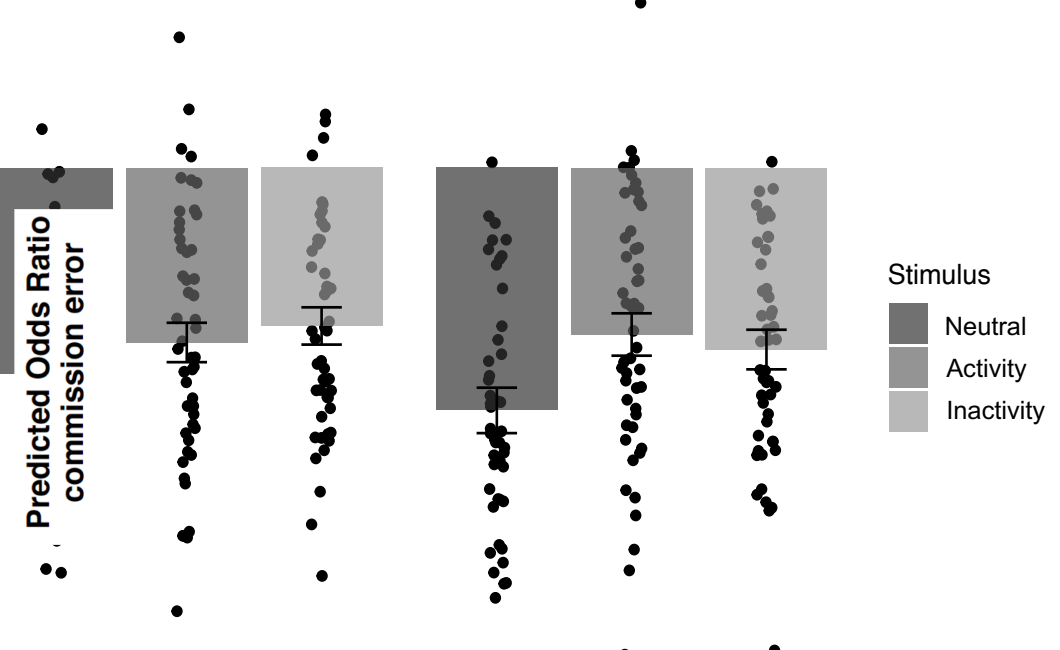

Note. Errors bars represent the $95 \%$ Confidence Interval around the mean. Dots represent the observations for each participant. 


\section{Figure 3}

N2 outcomes. A. Grand average ERPs and topoplot. The grand average ERPs are presented as a function of the type of trials and the type of stimuli, and the topoplot is presented averaged across type of trials and stimuli. Grand average ERPs reveal an N2 component peaking between 200 and $300 \mathrm{~ms}$. The topoplot demonstrates a stereotypical N2 frontocentral scalp distribution. B. N2 mean amplitudes. Data points representing mean amplitude for each participant as a function of the type of trial (i.e., go vs. no-go) and the type of stimuli (i.e., neutral vs. physical activity vs. physical inactivity).

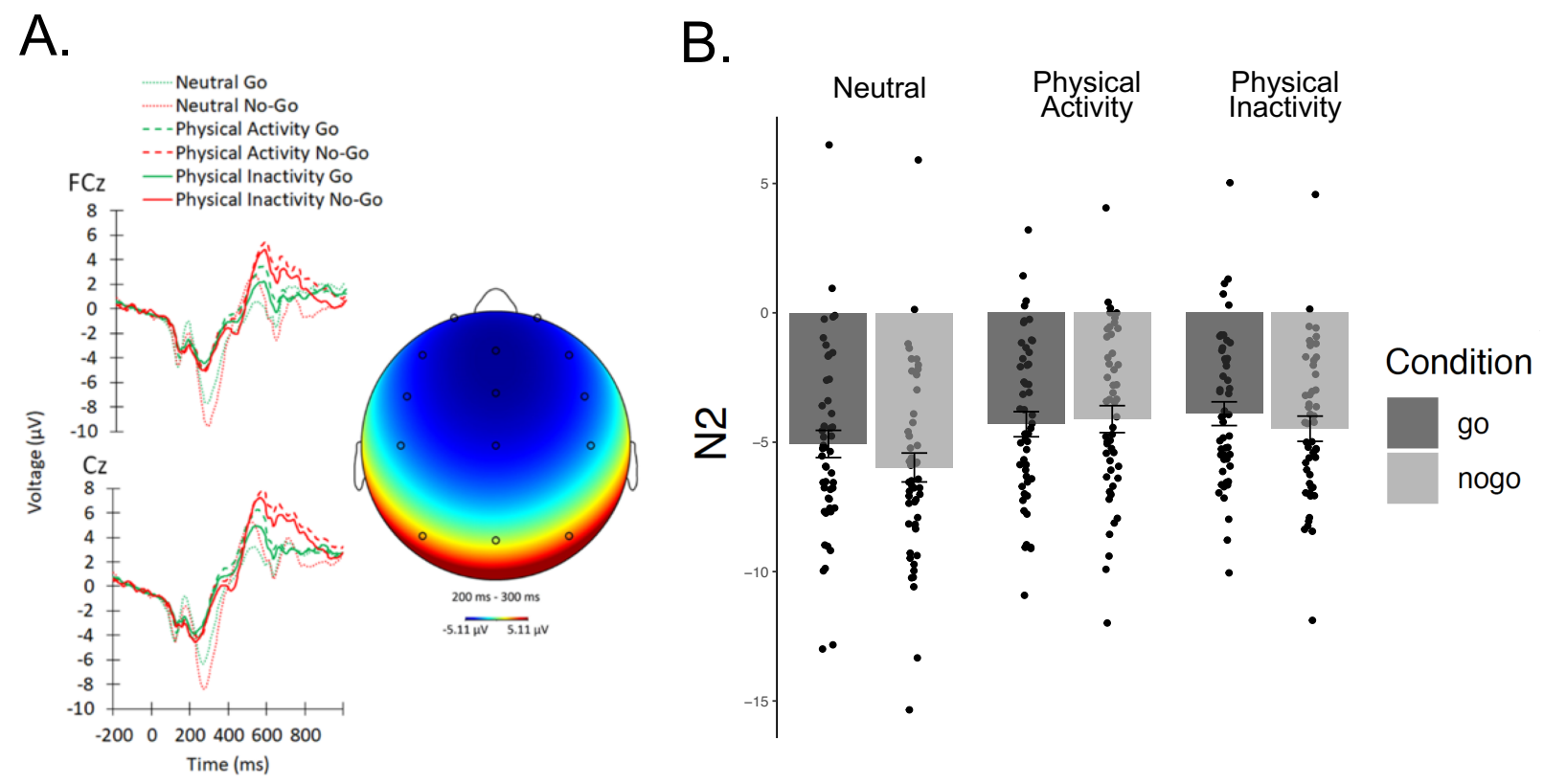

Note. Errors bars represent the $95 \%$ Confidence Interval around the mean. Dots represent the observations for each participant. 


\section{Figure 4}

Frontal Midline Theta (FMO) outcomes. A. Grand average time-frequency plots (FCz electrode). The grand average time-frequency plots are presented as a function of the type of trials and the type of stimuli, and the FM $\theta$ scalp distribution averaged across type of trial and stimuli is presented. Grand average time-frequency plots reveal low-frequency activity peaking just after $500 \mathrm{~ms}$. The topoplot of this activity reveals a stereotypical frontocentral scalp distribution. B. FM $\theta$ power. Data points representing FM $\theta$ power for each participant as a function of the type of trial (i.e., go vs. no-go) and the type of stimuli (i.e., neutral vs. physical activity vs. physical inactivity).

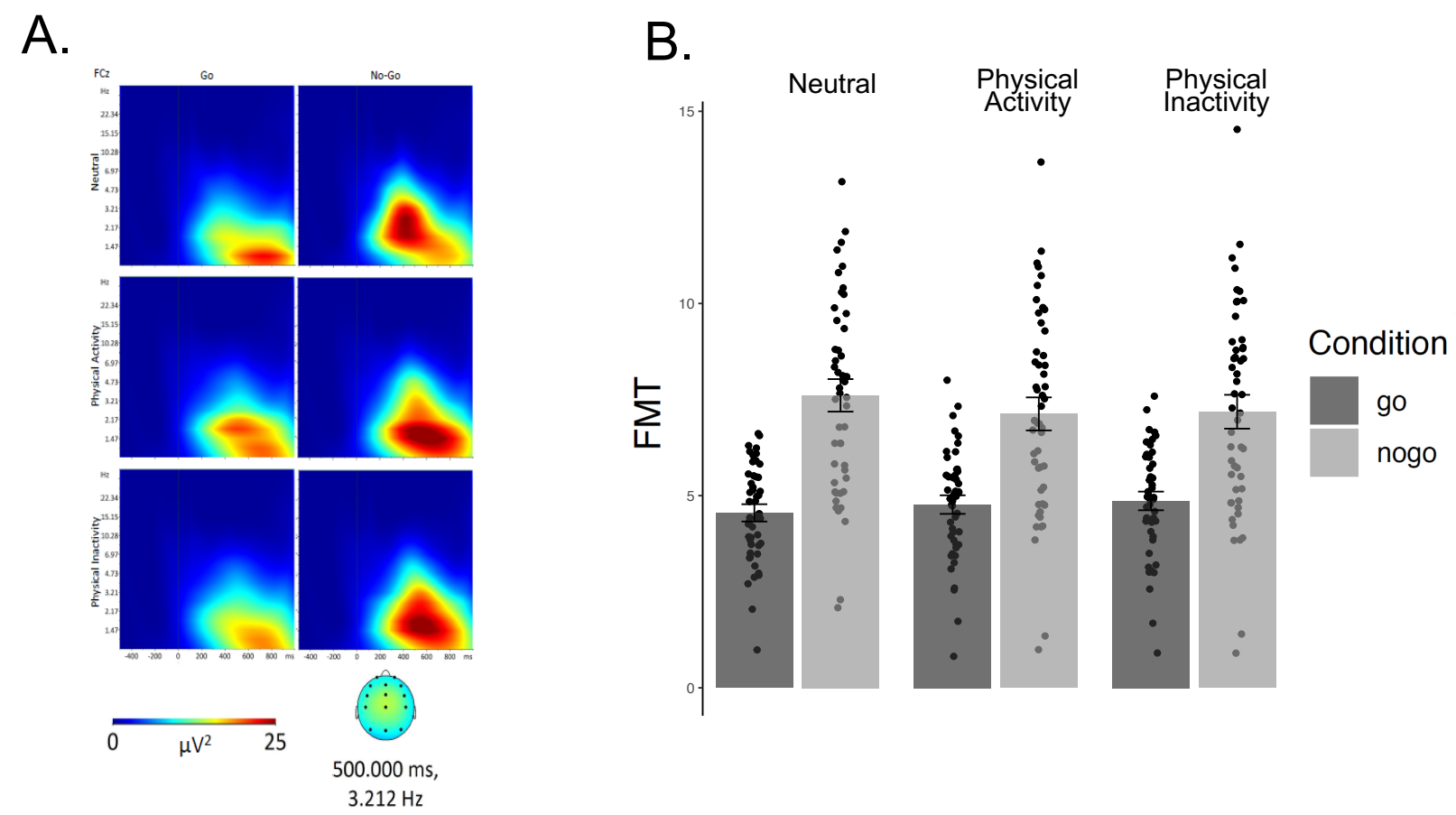

Note. Errors bars represent the 95\% Confidence Interval around the mean. Dots represent the observations for each participant. 